This is the final peer-reviewed accepted manuscript of:

A. Liguori [et al.]

Co-Deposition of Plasma-Polymerized Polyacrylic Acid and Silver Nanoparticles for the Production of Nanocomposite Coatings Using a NonEquilibrium Atmospheric Pressure Plasma Jet

in Plasma processes and polymers, vol.13, 6, 2016, pp.623-632

The final published version is available online at:

https://doi.org/10.1002/ppap.201500143

Rights / License:

This article may be used for non-commercial purposes in accordance with Wiley Terms and Conditions for Use of Self-Archived Versions. This article may not be enhanced, enriched or otherwise transformed into a derivative work, without express permission from Wiley or by statutory rights under applicable legislation. Copyright notices must not be removed, obscured or modified. The article must be linked to Wiley's version of record on Wiley Online Library and any embedding, framing or otherwise making available the article or pages thereof by third parties from platforms, services and websites other than Wiley Online Library must be prohibited."

This item was downloaded from IRIS Università di Bologna (https://cris.unibo.it/)

When citing, please refer to the published version. 


\title{
Co-Deposition of Plasma-Polymerized Polyacrylic Acid and Silver Nanoparticles for the Production of Nanocomposite Coatings Using a Non-Equilibrium Atmospheric
} Pressure Plasma Jet

\author{
Anna Liguori, Enrico Traldi, Elena Toccaceli, Romolo Laurita, \\ Antonino Pollicino, Maria Letizia Focarete, Vittorio Colombo, \\ Matteo Gherardi*
}

A single step process for the deposition of nanocomposite coatings with silver nanoparticles (AgNPs) embedded in a plasma-polymerized polyacrylic acid (pPAA) matrix and performed using a non-equilibrium atmospheric pressure plasma jet is presented. Acrylic acid (AA) and AgNPs dispersed in ethanol (EtOH) are used as precursors and are separately injected in the plasma region directly; $\mathrm{Ar}$ is used as plasma gas and also as carrier gas for both precursors. Scanning electron microscopy (SEM) and ATR-FTIR analysis show the deposition of a micrometric pPAA coating on the polyethylene (PE) film used as substrate; AgNPs embedded in the polymeric matrix are visible in SEM pictures and their presence is confirmed by XPS and EDS analysis. X-ray photoelectron spectroscopy (XPS) also highlights a high retention of carboxylic groups in the PPAA chemical structure and the surface oxidation of AgNPs. Preliminary results of the antibacterial activity of the co-deposited coatings are presented.

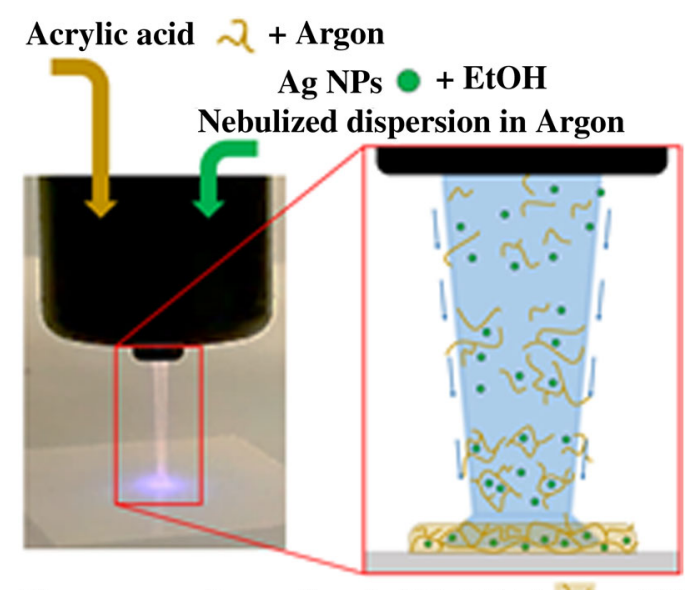

Nanocomposite coating AgNPs/pPAA on PE
A. Liguori, E. Traldi, E. Toccaceli, R. Laurita, V. Colombo, M. Gherardi Department of Industrial Engineering (DIN), Alma Mater Studiorum - Università di Bologna, Via Saragozza 8, 40123 Bologna, Italy

E-mail: matteo.gherardi4@unibo.it

A. Pollicino

Department of Industrial Engineering, Università di Catania, V.le A.Doria 6, 95125 Catania, Italy

M. L. Focarete

Department of Chemistry "Giacomo Ciamician", Alma Mater Studiorum - Università di Bologna, Via Selmi 2, 40126 Bologna, Italy

V. Colombo, M. Gherardi

Advanced Mechanics and Materials, Interdepartmental Center for Industri, al Research (AMM-ICIR), Alma Mater Studiorum -

Università di Bologna, Via Saragozza 8, 40123 Bologna, Italy

\section{Introduction}

Nanocomposite coatings, obtained embedding inorganic nanoparticles (NPs) into polymeric or inorganic matrices, have been raising great interest as innovative highperformance materials suitable for application in a wide range of industrial fields. ${ }^{[1-11]}$ Several techniques have been explored for the synthesis and deposition of nanocomposite coatings, spanning from chemical or electrochemical oxidation to plasma assisted processes. ${ }^{[12-19]}$

Focusing on non-equilibrium atmospheric pressure (cold) plasma technology, Fanelli et al. ${ }^{[20]}$ recently reviewed the state of the art of aerosol-assisted deposition processes and reported that while several studies have been dedicated 
to the deposition of polymeric and inorganic coatings, ${ }^{[21-32]}$ only a few have been focused on nanocomposite coatings. The first work on this topic was published in 2009 by Bardon et al. ${ }^{[33]}$ and reported on the deposition of a nanocomposite coating where $\mathrm{AlCeO}_{3} \mathrm{NPs}$ were embedded in an organosilicon matrix; the process was performed injecting in a Dielectric Barrier Discharge (DBD) reactor a NPs dispersion in a liquid organosilicon precursor. Later, Dembele et al. ${ }^{[34]}$ reported on the deposition of a nanocomposite coatings (organosilicon polymer and $\mathrm{TiO}_{2} \mathrm{NPs}$ ) obtained by introducing a dispersion of NPs in tetramethoxysilane in a plasma jet having a point to plane corona configuration. Some studies were also performed on the synthesis of organicinorganic nanocomposite coatings by means of atmospheric pressure cold plasma. In particular, Uygun et al. ${ }^{[35]}$ deposited different coatings by introducing a dispersion of polymer precursor (either pyrrole, thyophene, or furan monomers), $\mathrm{TiO}_{2} \mathrm{NPs}$, and $\mathrm{LiClO}_{4}$ in acetonitrile in an atmospheric pressure RF uniform glow discharge. Michel et al. ${ }^{[36]}$ investigated the deposition of plasma-polymerized polyaniline (PANI) coatings including mercaptoaniline functionalized PtNPs, by introducing a dispersion of functionalized NPs in ethanol and aniline in the plasma region of a DBD. Fanelli et al. ${ }^{[20]}$ reported on the deposition of ZnONPs/hydrocarbon polymer nanocomposite coatings using an atmospheric pressure DBD, fed with He and with an aerosol of oleate-capped ZnONPs dispersed in a hydrocarbon solvent.

Recently, cold plasma deposition of nanocomposite coatings with antibacterial properties has been investigated, exploiting the well known antimicrobial properties of AgNPs. ${ }^{[37]}$ Indeed, Beier et al. ${ }^{[38]}$ reported on a process where hexamethyldisiloxane, used as silicon containing precursor for the matrix, and a dispersion containing either $\mathrm{AgNO}_{3}$ or AgNPs were simultaneously injected in the plasma region of a DC pulsed arc discharge plasma jet. A different process, relying on the use of DC plasma jet, was proposed by Deng et al.; ${ }^{[39]} \mathrm{N}_{2}$ was used to sustain the plasma discharge and an admixture of $\mathrm{O}_{2}$, tetramethyldisiloxane, as organosilicon precursor for the matrix, $\mathrm{N}_{2}$, and AgNPs was fed to the plasma region. To date, only one work, published by Humud et al., ${ }^{[40]}$ proposed the fabrication of coatings containing AgNPs in a polymeric matrix using an atmospheric pressure plasma; the process, performed with a DBD plasma jet, was aimed at improving the conductivity of a polyaniline matrix by means of the introduction of AgNPs. As precursor, a dispersion of AgNPs in aniline was carried, in form of aerosol, by an Ar flow and injected into the plasma region. ${ }^{[40]}$

In this work, a single step process for the synthesis and deposition of nanocomposite coatings containing AgNPs embedded in a plasma-polymerized polyacrylic acid (pPAA) matrix, using a non-equilibrium atmospheric pressure dual gas plasma jet, ${ }^{[41-46]}$ is presented. The plasma jet, already employed with a nanopulsed generator for the deposition of pPAA coatings having a high retention of carboxylic groups, ${ }^{[41]}$ is here driven by a micropulsed generator, operated in Ar and fed with two precursors, AA for the matrix and a dispersion of AgNPs in EtOH, separately injected in the plasma region through the two gas channels of the plasma source. The coatings are deposited onto PE, a thermoplastic polymer widely employed in industrial and biomedical fields, with exceptionally easy processability features, good mechanical properties, and typically requiring surface modification to enhance its suitability for specific applications (e.g., increase of hydrophilicity for biomedical applications).

Results for the morphology, obtained by means of scanning electron microscopy (SEM), and chemical characteristics, obtained through attenuated total reflectance-fourier transform infrared spectroscopy (ATR-FTIR), Energy-dispersive X-ray spectroscopy (EDS), and X-ray photoelectron spectroscopy (XPS), of the nanocomposite coating are shown. Finally, the antibacterial efficacy of the deposited nanocomposite coatings is preliminary assessed against a test microorganism by means of agar disk diffusion tests.

\section{Experimental Section}

\subsection{Materials}

As a precursor for the nanocomposite coatings, 99\% anhydrous acrylic acid (AA) (Sigma-Aldrich, USA), for the fabrication of the polymeric matrix, and a dispersion $5 \% \mathrm{w} / \mathrm{w}$ of AgNPs (mean diameter $<100 \mathrm{~nm}$; Sigma-Aldrich) in anhydrous EtOH were used; the dispersion of AgNPs in EtOH was prepared by stirring the colloid at room temperature for $2 \mathrm{~min}$. Multi-layer films, composed of a polyethylene ( $\mathrm{PE}$, approximately $50 \mu \mathrm{m}$ thick), polyvinylidene chloride (PVDC, approximately $25 \mu \mathrm{m}$ thick), and polyvinylchloride (PVC, approximately $200 \mu \mathrm{m}$ thick) layers, were used as substrate; the co-deposition of the nanocomposite coatings of AgNPs embedded in a pPAA matrix (AgNPs/pPAA) was performed onto the PE layer. To support the discussion of SEM and EDS analyses, results will be also presented for PE films coated only with AgNPs, deposited using a dispersion of 5\% w/w of AgNPs in EtOH. In the text, nanocomposite coatings and nanoparticle coatings are referred to as AgNPs/pPAA coating and AgNPs coating, respectively.

\subsection{Non-Equilibrium Atmospheric Pressure Dual Gas Plasma Jet}

The plasma source adopted for the co-deposition of AgNPs/pPAA is a single electrode plasma jet, suitable for the treatment of different substrates such as metals, polymers, glasses, and biological materials, developed in our laboratory and reported in previous works. $^{[41-46]}$

As high-voltage electrode, a stainless steel sharpened metallic needle with a diameter of $0.3 \mathrm{~mm}$ is used; the plasma source is 
equipped with two gas conducts, to support the separate introduction of a primary and a secondary gases in the region surrounding the high-voltage electrode. The produced plasma is ejected from the source through an orifice with a diameter of $4 \mathrm{~mm}$, as described elsewhere. ${ }^{[41]}$

The plasma source was driven by a micropulsed generator producing high-voltage sinusoidal pulses having peak voltage $(P V)$ of up to $40 \mathrm{kV}$, frequency $(f)$ of $20-50 \mathrm{kHz}$, variable pulse duration, and fixed pulsed repetition frequency $(P R F)$ of $100 \mathrm{~Hz}$. During the plasma co-deposition process, $P V, f$ and duty cycle were kept constant at $23.4 \mathrm{kV}, 20 \mathrm{kHz}$, and $40 \%$, respectively.

\subsection{Experimental Setup}

The experimental setup is schematically represented in Figure 1. For the AgNPs/pPAA deposition onto PE substrates, the plasma jet was operated in Ar and separately fed with the nanocomposite coating precursors, exploiting the two distinct gas channels of the plasma source. In particular, a flow of $2.5 \mathrm{slpm}$ of Ar was introduced at first inside a bubbler containing the monomer and then, carrying the AA, injected into the plasma source through the primary channel, as described in our previous work. ${ }^{[41]}$ Simultaneously, a second flow of $2.5 \mathrm{slpm}$ of Ar was introduced in a nebulizer system containing the dispersion of AgNPs in EtOH and the so formed aerosol was injected into the plasma source through the secondary gas channel. The mass flow rate of AA injected through the primary channel and of the AgNPs nebulized dispersion injected through the secondary channel, determined by the monomer and colloid consumption inside the flasks as reported in previous works, ${ }^{[47,48]}$ were of $0.05 \mathrm{ml} / \mathrm{min}$ and $2.3 \mathrm{ml} / \mathrm{min}$, respectively. For the deposition of AgNPs coatings, the primary gas channel was closed and only the aerosol of AgNPs in Ar was fed to the plasma region. During the deposition process, the distance between the plasma source and the PE substrate was kept constant at $2 \mathrm{~mm}$ and a floating aluminum foil was placed under the PE film in order to facilitate the generation of the plasma discharge. For all the experiments, the treatment time was kept constant at $3 \mathrm{~min}$.

\subsection{Characterization of the Co-Deposited AgNPs/ pPAA Nanocomposite Coating}

\subsubsection{Scanning Electron Microscopy}

Scanning electron microscopy was performed to investigate the morphology of the Ag NPs/pPAA coatings deposited onto PE substrates; for comparison, SEM analysis was also performed on substrates where only AgNPs were deposited. Scanning Electron Microscope observations were carried out using a Philips 515 SEM by applying an accelerating voltage of $15 \mathrm{kV}$, on samples sputtercoated with gold; the SEM was also equipped with energy dispersive X-ray spectroscopy (EDS), which was used for elemental analyses of the samples.

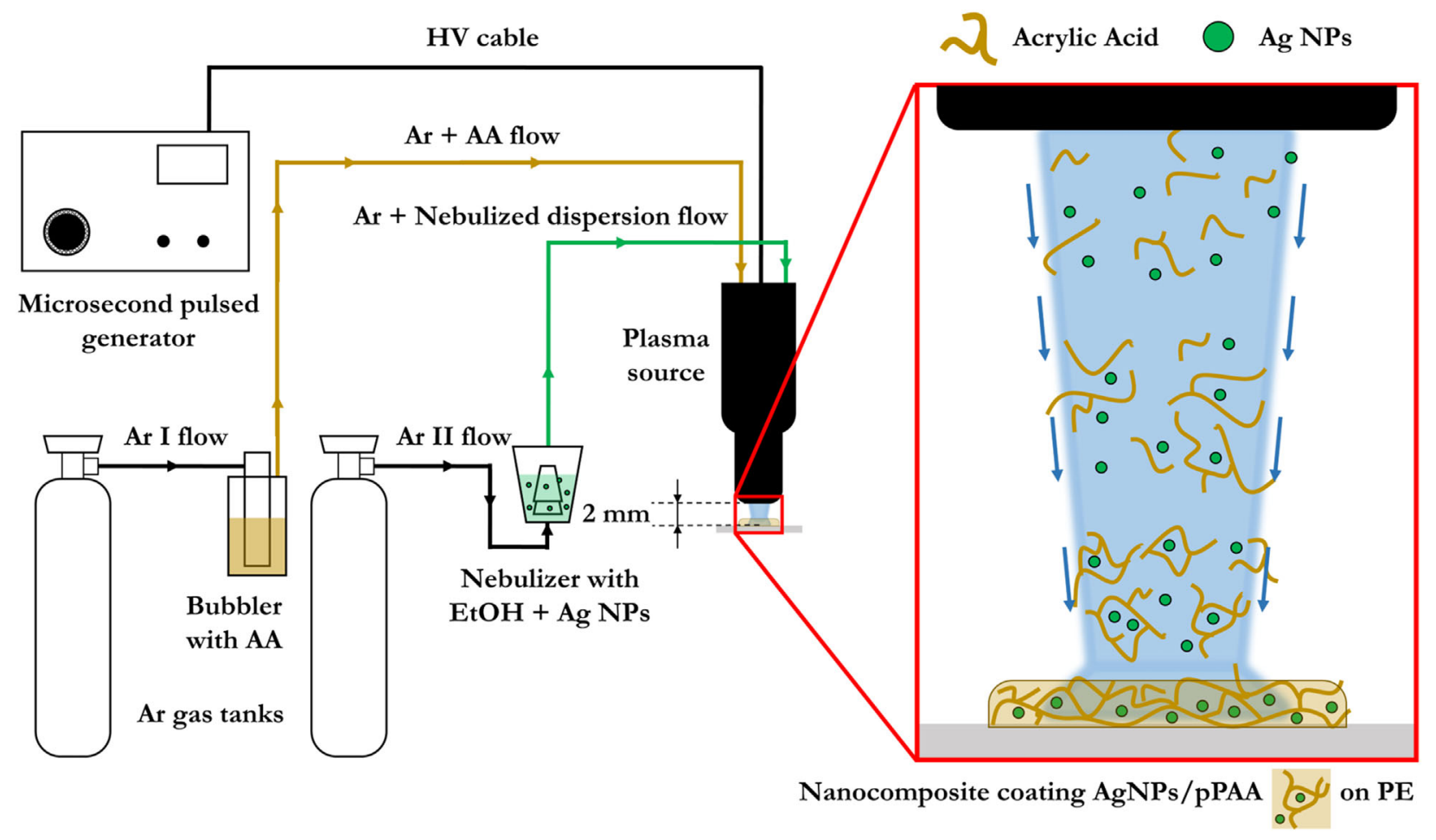

Figure 1. Experimental setup of the plasma co-deposition process. 


\subsubsection{Attenuated Total Reflectance-Fourier Transform Infrared Spectroscopy}

Attenuated total reflectance-fourier transform infrared (Agilent Cary 660 FTIR spectrometer) was used to gather information on the chemical structure of the substrate before and after the deposition of the AgNPs/pPAA coatings. The spectrometer was equipped with an ATR sampling device, using a diamond crystal as internal reflection element. Infrared spectra were acquired at room temperature (RT) in absorbance mode, from 3900 to $400 \mathrm{~cm}^{-1}$ with a resolution of $2 \mathrm{~cm}^{-1}$; a total of 32 scans were recorded for each spectrum.

\subsubsection{X-Ray Photoelectron Spectroscopy}

$\mathrm{X}$-ray photoelectron spectroscopy measurements were carried out on a VG Instrument electron spectrometer using a $M g \mathrm{~K}_{\alpha 1,2}$ Xray source $(1253.6 \mathrm{eV})$. The X-ray source in the standard conditions had been working at $300 \mathrm{~W}, 15 \mathrm{kV}$, and $20 \mathrm{~mA}$. The base pressure of the instrument was $5 \times 10^{-10}$ Torr and an operating pressure of $2 \times 10^{-8}$ Torr was adopted. A pass energy of $100 \mathrm{eV}$ and $20 \mathrm{eV}$ was used for widescans and narrowscans, respectively. The semiquantitative surface analyses were carried out by the determination of the photoelectron peak areas obtained by multiplying the experimental values with the appropriate sensitivity factor. For acquiring the spectra, a take-off angle of $80^{\circ}$ was used. Considering that the relationship between the depth of the analyzed layer (d) and the t.o.a. $(\theta)$ is represented by the equation $d=3 \lambda \sin \theta$, where $\lambda$ is the inelastic mean free path (IMFP) of the photoelectrons (for carbon $\lambda=14 \AA$ ), ${ }^{[4]}$ it is possible to quantify the thickness of the analyzed layer in about $40 \AA$. The calculation of the areas corresponding to the different photoelectron peaks was performed using VGX900x software; the curve fitting elaborations were done by means of PeakFit software (version 4, from SPSS Inc.). The curve fitting of $C_{1 s}$ envelope has been performed using the product of Gaussian and Lorentzian functions (80:20): the FWHM of the height of each curve was kept equal to $1.7 \pm 0.1 \mathrm{eV}$. Binding energies were referred to the $\mathrm{C}-\mathrm{H}$ level at $285 \mathrm{eV}$. presence/absence of a bacterial growth inhibition area was evaluated. Tests were performed in triplicate.

\section{Results and Discussion}

Thickness and morphology of the AgNPs/pPAA coatings were investigated by means of SEM analysis. In particular, the coating thickness was measured to be around $30 \mu \mathrm{m}$ from SEM images of the cross-sectional view of the co-deposited samples; a representative crosssectional view of a sample coated with AgNPs/pPAA is shown in Figure 2.

This measured thickness is significantly higher than those achieved with other atmospheric pressure plasma sources. ${ }^{[31,32,50,51]}$ Indeed, Vogelsang et al., ${ }^{[31]}$ in their work on the deposition of C:F coatings using a RF capillary microplasma jet, reported of a maximum coating thickness of $7.8 \mu \mathrm{m}$ after $3 \mathrm{~min}$ of deposition; Chen et al., ${ }^{\left[{ }^{32]}\right.}$ reporting on the deposition of pPAA coatings with an atmospheric pressure glow discharge plasma jet, observed a thickness of around $300 \mathrm{~nm}$ after a $10 \mathrm{~min}$ deposition time. Bashir et al., ${ }^{[50]}$ in their work on the polymerization of hexamethyldisiloxane using a DBD plasma jet, reported about an average thickness of the deposited coating of $662 \pm 33 \mathrm{~nm}$ after a treatment time of $3 \mathrm{~min}$. Finally, Bosso et al. ${ }^{[51]}$ observed a maximum thickness of $300 \mathrm{~nm}$ for a pPAA coating deposited by means of DBD plasma jet (10 min deposition time).

Scanning electron microscopy top views of the samples, reported in Figure 3, show that the co-deposited coating is characterized by an uneven surface, with bright regions in correspondence of polymer-coated AgNPs and aggregates (Figure $3 b, d$, and f) similarly to what previously reported by Deng et al.; ${ }^{[39]}$ the dimension of these bright areas are almost coincident with the

\subsection{Antimicrobial Assay}

Escherichia coli (DSM 3083) was cultured on Tryptic soy agar (TSA) plates at $37^{\circ} \mathrm{C}$ and a bacterial suspension was prepared in sterile distilled water from an overnight culture; the suspension was adjusted to approximately $1.5 \times 10^{8} \mathrm{CFU}$ (Colony Forming Units) $\mathrm{ml}^{-1}$ based on McFarland turbidity standards, serially diluted and plated on TSA to quantify the bacterial concentration. The suspension was spread over the entire surface of the TSA plate by swabbing uniformly across agar. Each plate was kept for $15 \mathrm{~min}$ at room temperature and then each sample to be tested (either pristine PE, PE coated with pPAA or PE coated with AgNPs/pPAA), having a surface $1 \mathrm{~cm}^{2}$, was placed onto a contaminated agar plates. After incubation for $24 \mathrm{~h}$ at $37^{\circ} \mathrm{C}$, the
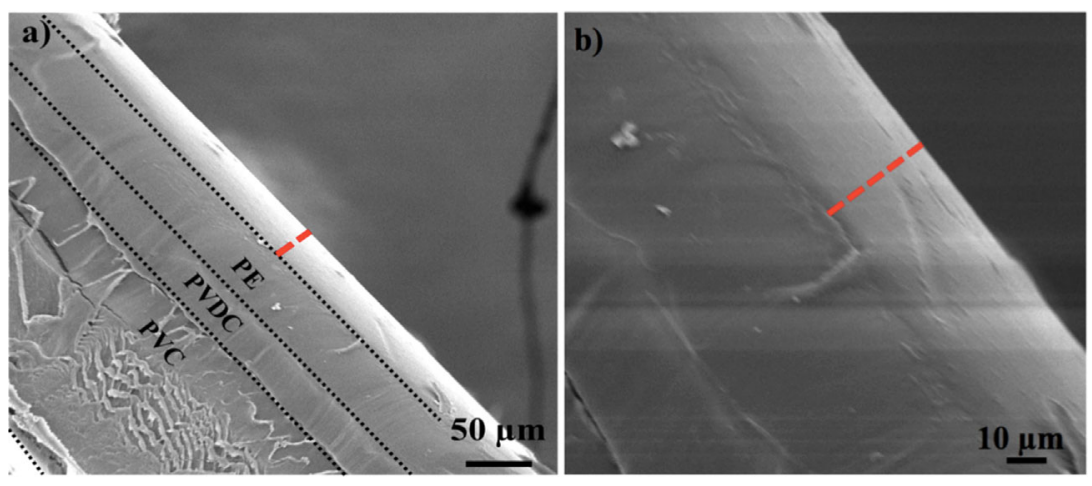

Figure 2. SEM images of the cross-sectional view of the AgNPs/pPAA coated PE; the coating thickness is indicated by the broken red line. Dotted black lines are drawn in (a) in correspondence to the boundaries of the polymeric layers constituting the multilayer film used as substrate. Magnification: $220 \times(a), 810 \times(b)$. 

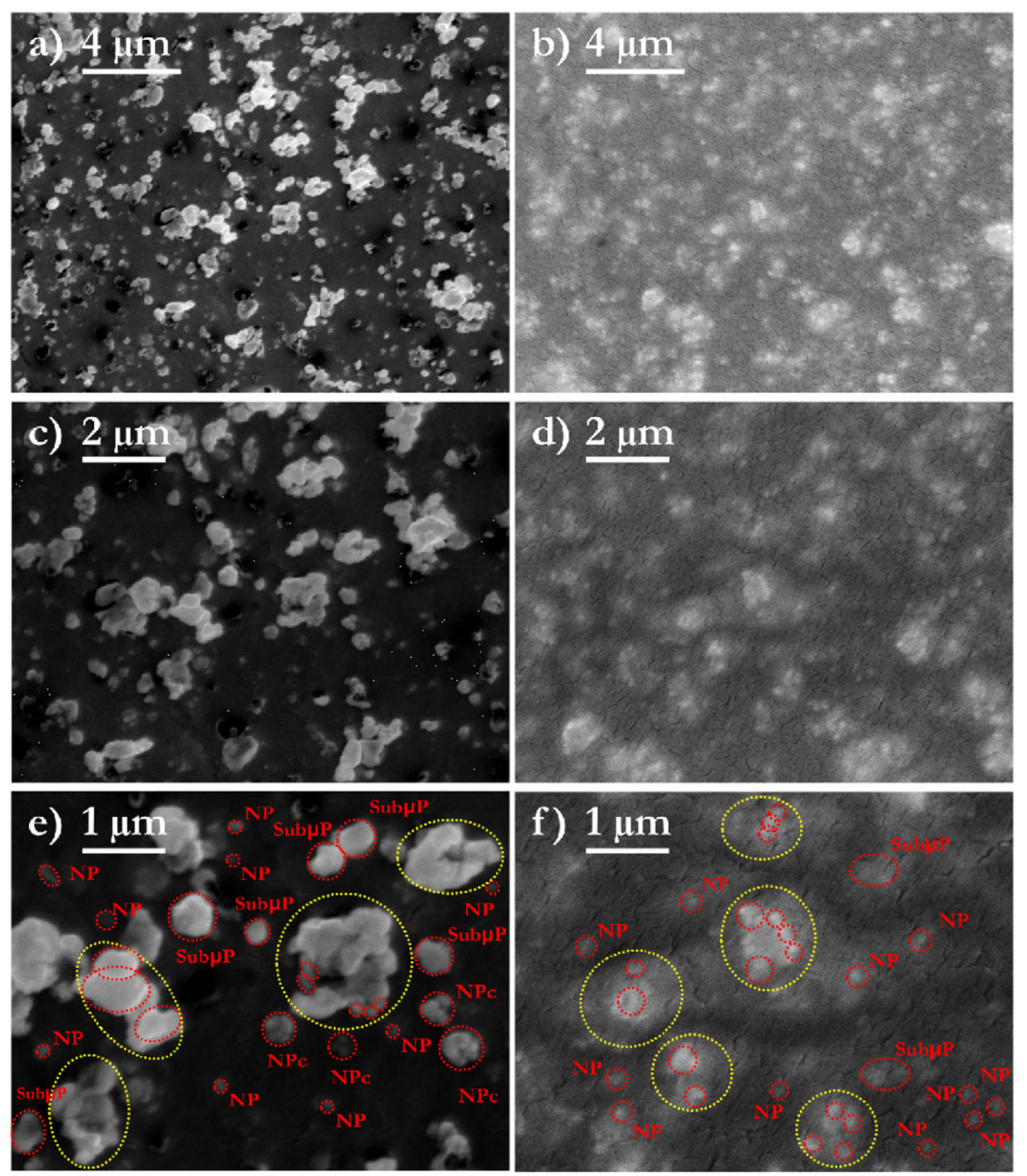

Figure 3. SEM images of the top views of the PE films coated with AgNPs (a, c, and e) and AgNPs/pPAA (b, d, and f). Magnification: $6000 \times(a$ and b); $10000 \times(c$ and d), $20000 \times$ (e and f). Dotted yellow circles indicate micrometric aggregates; dotted red circles indicate AgNPs with characteristic dimension around $100 \mathrm{~nm}$, either isolated (NP) or organized in clusters (NPc), and Ag sub-micrometric particles with characteristic dimension in the range of few hundreds nanometres (Sub $\mu \mathrm{P})$.

dimension of AgNPs particles and aggregates that can be observed on samples obtained by depositing only AgNPs (Figure 3a, c, and e). The highest resolution SEM top views (Figure $3 e$ and $\mathrm{f}$ ) underline the presence, in both the AgNPs/pPAA and AgNPs coatings, of AgNPs (having characteristic dimension around $100 \mathrm{~nm}$ ) either isolated (marked as NP in Figure 3e and f) or organized in clusters (marked as NPc in Figure 3e and f) and sub-micrometric particles (having characteristic dimension of few hundreds of nanometers and marked as sub $\mu$ P in Figure $3 e$ and f); micrometric aggregates (circled by a broken yellow line in Figure $3 e$ and f) are visible as well in the coating. The aggregation of nanoparticles during the co-deposition process was reported also by Deng et al. ${ }^{[39]}$
The presence of $\mathrm{Ag}$ in the AgNPs/ pPAA coatings is further confirmed by the collected EDS spectra shown in Figure 4; noticeable, the Ag characteristic peak is slightly lower in the AgNPs/pPAA coating than in the AgNPs one, as a consequence of the AgNPs being embedded in the polymeric matrix.

Attenuated total reflectance-fourier transform infrared spectroscopy analysis confirmed the successful deposition on the PE substrate of the PPAA matrix of the AgNPs/pPAA coating, as the collected spectrum, shown in Figure 5, is remarkably similar to other pPAA spectra previously reported in literature. ${ }^{[41,51]}$ Despite some works have indicated that ATR-FTIR might provide information about the molecular environment of the organic molecules on the surface of AgNPs ${ }^{[52,53]}$ and thus lead to an indirect detection of the $\mathrm{Ag}$ in the coating through a slight shift of characteristic peaks of the spectrum, ${ }^{[40]}$ we could not observe any significant difference between AgNPs/pPAA and a typical pPPA coating, probably because the amount of Ag in our coating is below the sensitivity threshold of the technique.

The XPS survey spectrum of the AgNPs/pPAA coating, reported in Figure 6 , highlighted the presence of $\mathrm{Ag}, \mathrm{C}, \mathrm{O}$, and $\mathrm{N}$ through their corresponding XPS peaks $\left(\mathrm{C}_{1 \mathrm{~s}}, \mathrm{O}_{1 \mathrm{~s}}, \mathrm{~N}_{1 \mathrm{~s}}, \mathrm{Ag}_{3 \mathrm{~d}}\right.$, and $\mathrm{Ag}(\mathrm{A}))$. The elemental concentrations in the nanocomposite coating, jointly with the atomic ratios, are reported in Table 1.

The presence of nitrogen in the XPS survey spectrum can be probably appointed to the interaction of AA, or the products of its plasma-polymerization, with vibrationally excited $\mathrm{N}_{2}$ molecules, resulting from the mixing of the plasma plume with the surrounding air, ${ }^{[43]}$ as proposed by Bhatt et al. ${ }^{[54]}$ for the case of plasmapolymerization of diethylene glycol dimethyl ether; indeed, they observed by means of optical emission spectroscopy the formation of $\mathrm{OH}, \mathrm{CH}, \mathrm{N}_{2}$, and $\mathrm{CN}$ excited species when the monomer was introduced into the Ar plasma jet.

The curve fitting of the high-resolution $\mathrm{C}_{1 \mathrm{~s}}$ peak, shown in Figure 7, provides useful information on the retention of carboxyl groups in the chemical structure of the pPAA 


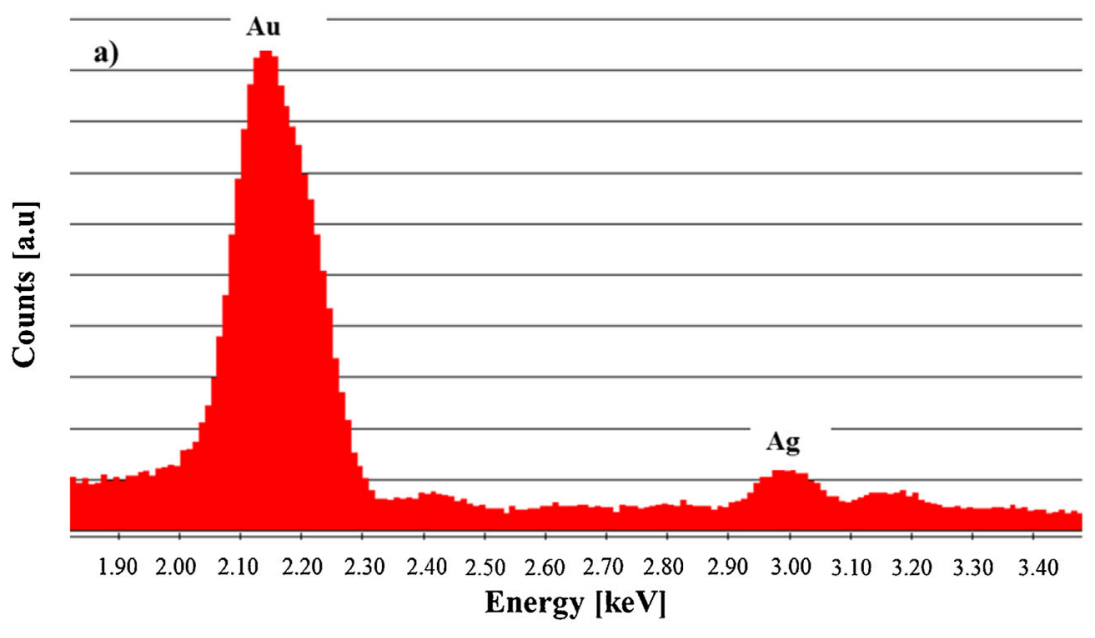

b)

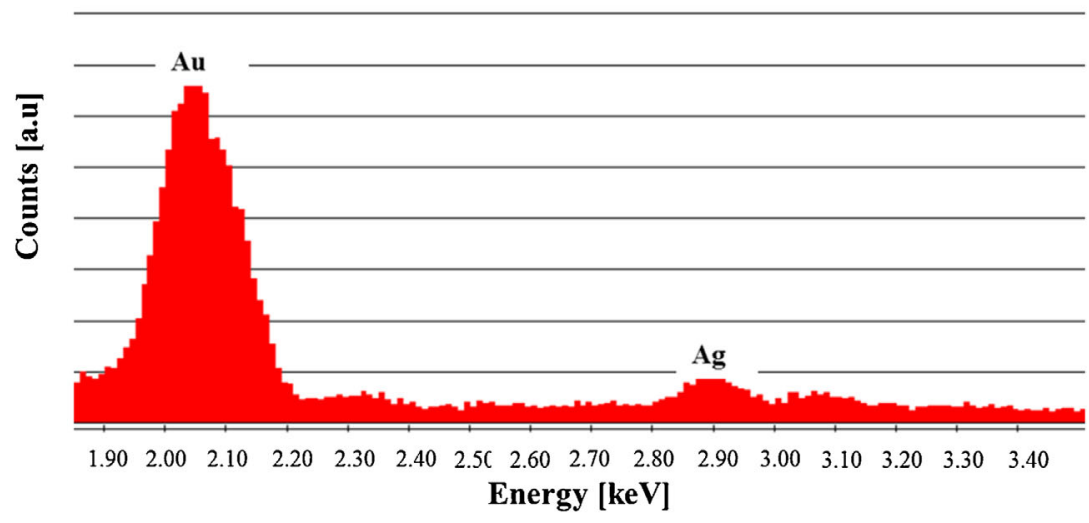

Figure 4. EDS spectra of PE films coated with AgNPs (a) and AgNPs/pPAA (b). Samples were sputter-coated with gold prior to examination.

matrix of the nanocomposite coating. Indeed, the $C_{1 s}$ envelope of the deposited nanocomposite coating can be deconvoluted into four distinct peaks: a peak at $285.0 \pm 0.1 \mathrm{eV}$ corresponding to $\underline{\mathrm{C}} \mathrm{C}$ and $\underline{\mathrm{C}}-\mathrm{H}$ bonds, $\mathrm{a}$

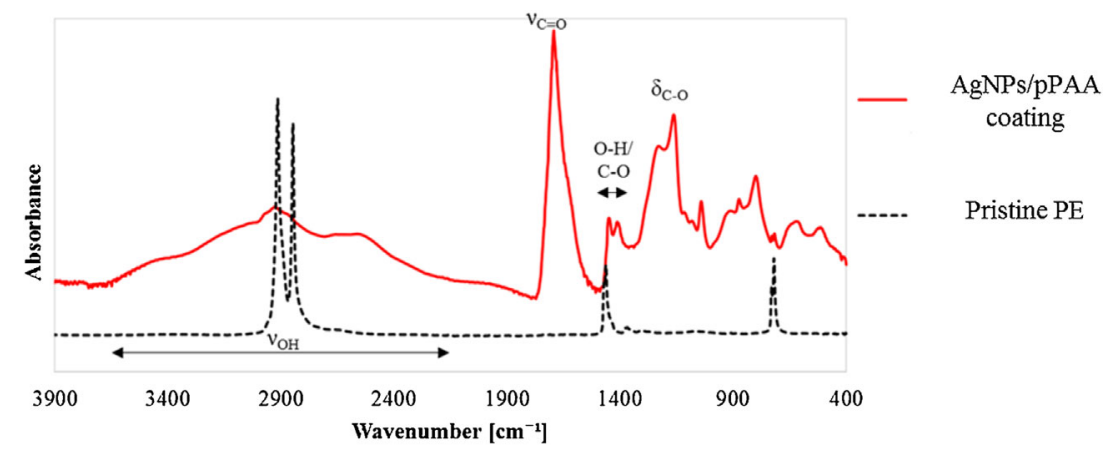

Figure 5. ATR-FTIR spectra of uncoated PE and of AgNPs/pPAA coated PE. peak at $285.5 \pm 0.1 \mathrm{eV}$ due to $\underline{\mathrm{C}}-\mathrm{COOH}$ functional groups, a peak at $287.8 \pm 0.1 \mathrm{eV}$ due to $\mathrm{C}=\mathrm{O}$ bonds and, finally, a peak at $289.1 \pm 0.1 \mathrm{eV}$, attributed to carboxylic acid $(-\underline{\mathrm{COOH}})$ and/or ester (- $\underline{\text { COOR) }}$ groups.

Interestingly, as reported in Table 2, the AA plasma-polymerization carried out by means of the micropulsed nonequilibrium atmospheric pressure plasma jet leads to a high retention of $-\mathrm{COOH} / \mathrm{R}$ groups (21\%); this amount is fairly close to the one previously measured for pPAA deposited with the same plasma source, but driven by a nanosecond pulsed generator. ${ }^{[41]}$ This result can be probably attributed to the low value of duty cycle employed for the co-deposition process: in fact, as highlighted by Boscher et al. ${ }^{[55]}$ in their work on the deposition of plasma-polymerized poly(glycidyl methacrylate) by means of DBD driven by ultra-short square pulses, the increase of the duty cycle leads to functional groups destruction and to the formation of a larger distribution of chemical bonds; conversely, films deposited using lower duty cycle conditions were found to be identical in terms of chemical composition to the ones obtained for conventionally polymerized poly(glycidyl methacrylate). ${ }^{[55]}$

High-resolution XPS spectra of the $\mathrm{Ag}_{3 \mathrm{~d}}$ are reported in Figure 8 and indicate that the binding energies of the corresponding spin orbit splitting of $\mathrm{Ag}_{3 \mathrm{~d} 5 / 2}$ and $\mathrm{Ag}_{3 \mathrm{~d} 3 / 2}$ due to AgNPs are centered at $368.2 \mathrm{eV}$ and $374.2 \mathrm{eV}$, respectively. Nonetheless, the binding energy positions of $\mathrm{Ag}_{3 \mathrm{~d}}$ were not enough to identify the oxidation state of the $\mathrm{Ag}$ species because the characteristic states of oxidized and metallic silver are close together. ${ }^{[56,57]}$ Nonetheless, partially oxidized AgNPs are known to exhibit better antibacterial activities than the zero-valent $\mathrm{Ag}^{[58]}$ thus, the kinetic energy (KE) in the Ag MNN region of the Auger transitions was measured and the modified Auger parameter $\left(\alpha^{\prime}\right)$ was used to characterize the chemical state of Ag. This parameter, originally proposed by Wagner ${ }^{[59,60]}$ and 


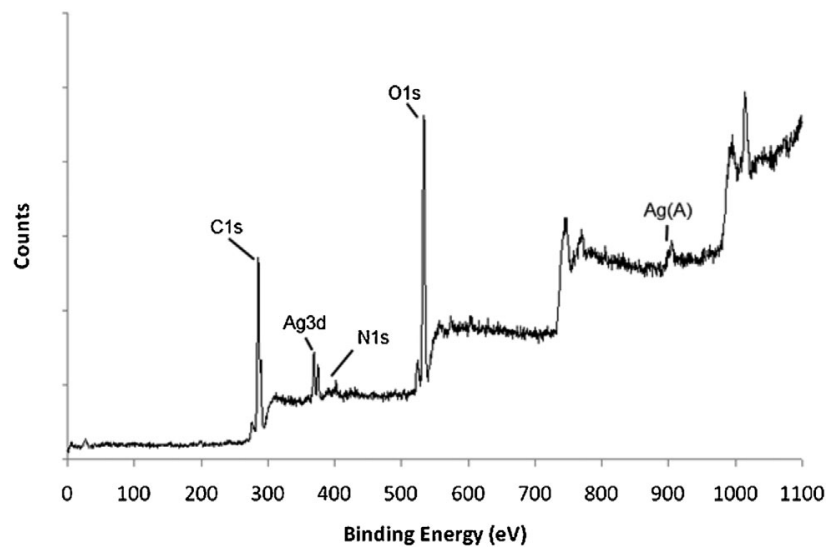

Figure 6. XPS survey spectrum collected for the AgNPs/pPAA coating.

Table 1. Elemental concentration and atomic ratios measured for the AgNPs/pPAA coating.

\begin{tabular}{lcccccc}
\hline $\mathbf{C}$ & $\mathbf{O}(\%)$ & $\mathbf{N}(\%)$ & $\mathbf{A g}(\%)$ & $\mathbf{O} / \mathbf{C}$ & $\mathbf{N} / \mathbf{C}$ & $\mathbf{A g} / \mathbf{C}$ \\
\hline $63.2 \%$ & 34.2 & 1.7 & 0.9 & 0.54 & 0.026 & 0.014 \\
\hline
\end{tabular}

successively modified by Gaarenstrom et al., ${ }^{[61]}$ is the sum of the kinetic energy of the Auger electron (in our case Ag $\left.M_{4} \mathrm{~N}_{4,5} \mathrm{~N}_{4,5}\right)$ and the binding energy of the corelevel $\left(\mathrm{Ag}_{3 \mathrm{~d} 5 / 2}\right)$ peak. ${ }^{[62]}$ This parameter was independent of the charging, but still sensitive to the chemical state

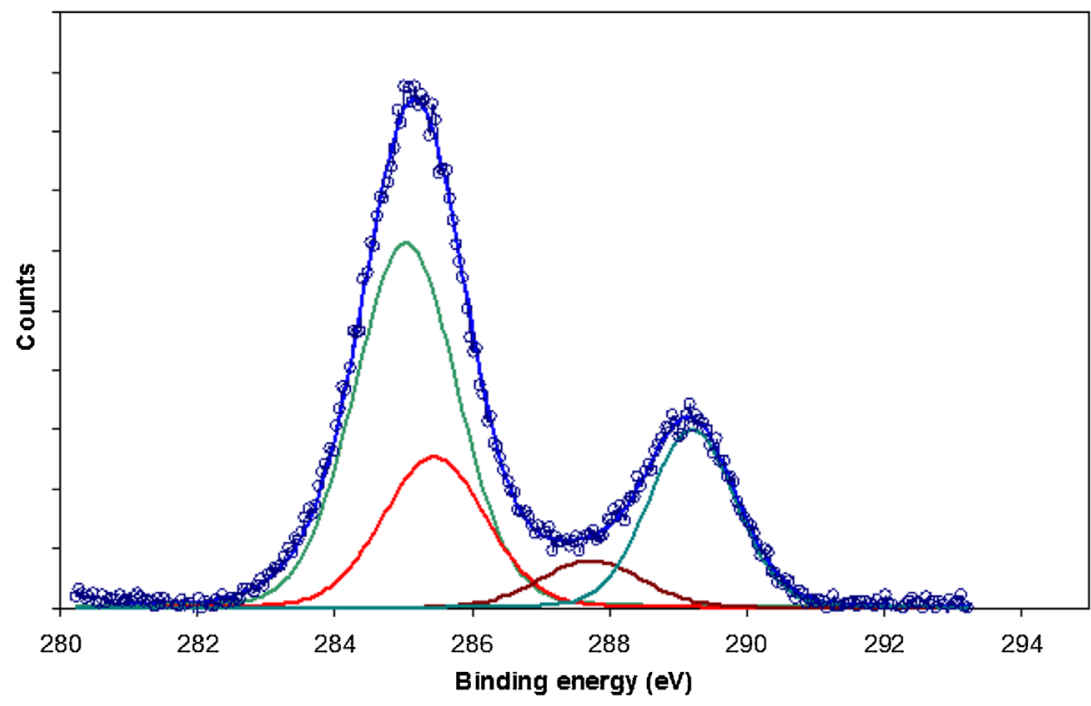

Figure 7. XPS deconvoluted $C_{15}$ peak of the AgNPs/pPAA coating.
Table 2. Surface carbon groups concentration of the AgNPs/pPAA coating.

\begin{tabular}{lccc}
\hline $\mathbf{C}-\mathbf{c}$ \\
$\mathbf{C}-\mathbf{H}$ \\
$285.0 \mathrm{eV}$ & $\underline{\mathbf{c}}-\mathbf{C O O H}$ & $\mathbf{C}=\mathbf{0}$ & $\begin{array}{l}-\mathbf{C O O H} \\
-\mathbf{C O O R}\end{array}$ \\
\hline $52 \%$ & $285.5 \mathrm{eV}$ & $287.8 \mathrm{eV}$ & $289.1 \mathrm{eV}$ \\
\hline
\end{tabular}

of silver and it was calculated according to the Equation (1):

$$
\alpha^{\prime}(\mathrm{eV})=\mathrm{KE}\left(\mathrm{AgM}_{4} \mathrm{~N}_{4,5} \mathrm{~N}_{4,5}\right)-\mathrm{KE}\left(\mathrm{Ag}_{3 \mathrm{~d} 5 / 2}\right)+\mathrm{h} v
$$

where $\mathrm{KE}$ ( $\mathrm{Ag} \mathrm{M}_{4} \mathrm{~N}_{4,5} \mathrm{~N}_{4,5}$ ) is the kinetic energy of the Auger transition, $\mathrm{KE}\left(\mathrm{Ag}_{3 \mathrm{~d} 5 / 2}\right)$ is the kinetic energy of the $\mathrm{Ag}_{3 \mathrm{~d} 5 / 2}$ core-level and $\mathrm{h} v$ is the photon energy equal to $1253.6 \mathrm{eV}$.

The AgNPs/pPAA coated samples showed a photoelectron peak Ag3d $\mathrm{d}_{5 / 2}$ centered at $368.2 \mathrm{eV}$, and the $\mathrm{M}_{4} \mathrm{~N}_{4,5} \mathrm{~N}_{4,5}$ Auger peak centered at a kinetic energy equal to $355.3 \mathrm{eV}$. The $\alpha^{\prime}$ parameter is, therefore, equal $355.3+368.2=723.5$. Based on the values reported by NIST Database and Wagner, ${ }^{[57,63]}$ this parameter value can be associated with the presence of AgO.

As a last consideration on XPS results, it is worth reminding that XPS analysis provides information on the atomic abundance only at the outermost $4 \mathrm{~nm}$ of the coating. Since the characteristic dimension of the single AgNP or cluster encapsulated in the polymeric coating is higher than $100 \mathrm{~nm}$, the $\mathrm{Ag}$ concentration detected by the surface analysis performed with the XPS cannot be considered as indicative of the bulk chemical composition of the coating, but it is expected to underestimate the $\mathrm{Ag}$ content as mentioned in previous works. ${ }^{[64]}$

Results of agar disk diffusion tests to evaluate the antibacterial efficiency of the coatings are shown in Figure 9: while no growth inhibition area can be observed around the uncoated PE and PPAA coated PE samples, a clear zone with no bacterial growth can be clearly detected around the AgNPs/pPAA coated PE samples, similarly to what previously reported also by Sadeghnejad et al.; ${ }^{[65]}$ this behavior is indicative of the action Ag ions, probably released from 

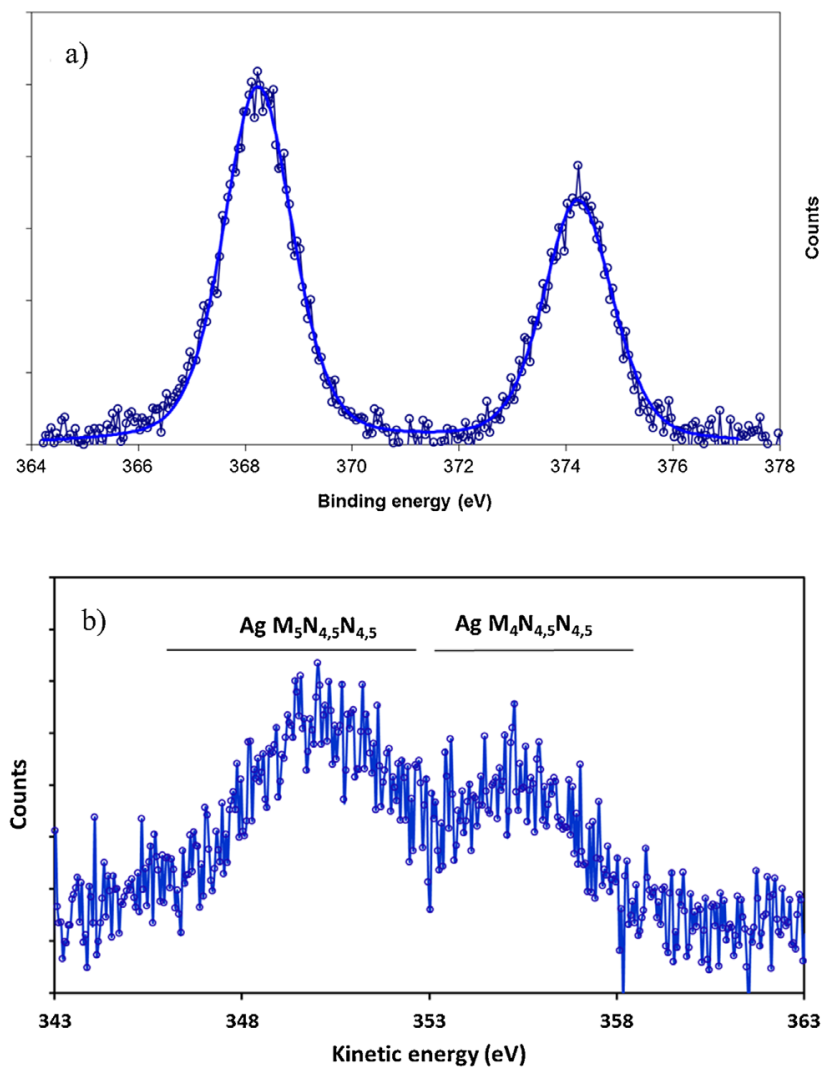

Figure 8. $\mathrm{Ag}_{3 \mathrm{~d}}$ core-level (a) and $\mathrm{Ag}$ MNN Auger transition spectra (b) of the AgNPs/pPAA coating.

the Ag present either at the surface of the AgNPs/ pPAA coating or made available for oxidation by some cracks in the coating (that can be seen quite clearly in Figure $3 \mathrm{~d}$ and $\mathrm{f}$ ), similarly to what previously proposed in literature. ${ }^{[39,66,67]}$

\section{Conclusion}

In the present work, a single step plasma process for the synthesis and the deposition of coatings containing AgNPs embedded in a pPAA matrix has been reported. The process was performed using a nonequilibrium atmospheric pressure plasma jet enabling the separate and simultaneous introduction of AA and AgNPs dispersion in the plasma region. Furthermore, a thorough morphological and chemical characterization of the co-deposited coatings has been here reported. The thickness of AgNPs/pPAA coatings co-deposited for $3 \mathrm{~min}$ was measured to be around $30 \mu \mathrm{m}$ from SEM cross-sectional views of the samples. attenuated total reflectance-fourier transform infrared spectroscopy spectrum of the co-deposited AgNPs/pPAA coating was found to coincide with the spectrum of pPAA coating without AgNps embedded. X-ray photoelectron spectroscopy results highlighted a significant (21\%) retention of carboxylic groups in the pPAA chemical structure, underlining that limited monomer fragmentation occurred during the process, ${ }^{[41]}$ and the presence in the coating of superficially oxidized AgNPs. Furthermore, the antibacterial efficacy of the co-deposited AgNPs/pPAA coatings was preliminary assessed with agar disk diffusion tests (using E. coli) and a growth inhibition area surrounding the samples, due to the release of $\mathrm{Ag}$ ions from the pPAA matrix, was clearly visible after incubation for $24 \mathrm{~h}$ at $37^{\circ} \mathrm{C}$.

Future studies will be aimed at evaluating the time evolution of the morphological and chemical characteristics and antibacterial properties in different environmental conditions of such co-deposited coatings, also taking into account their possible use for different applications in the biomedical field.
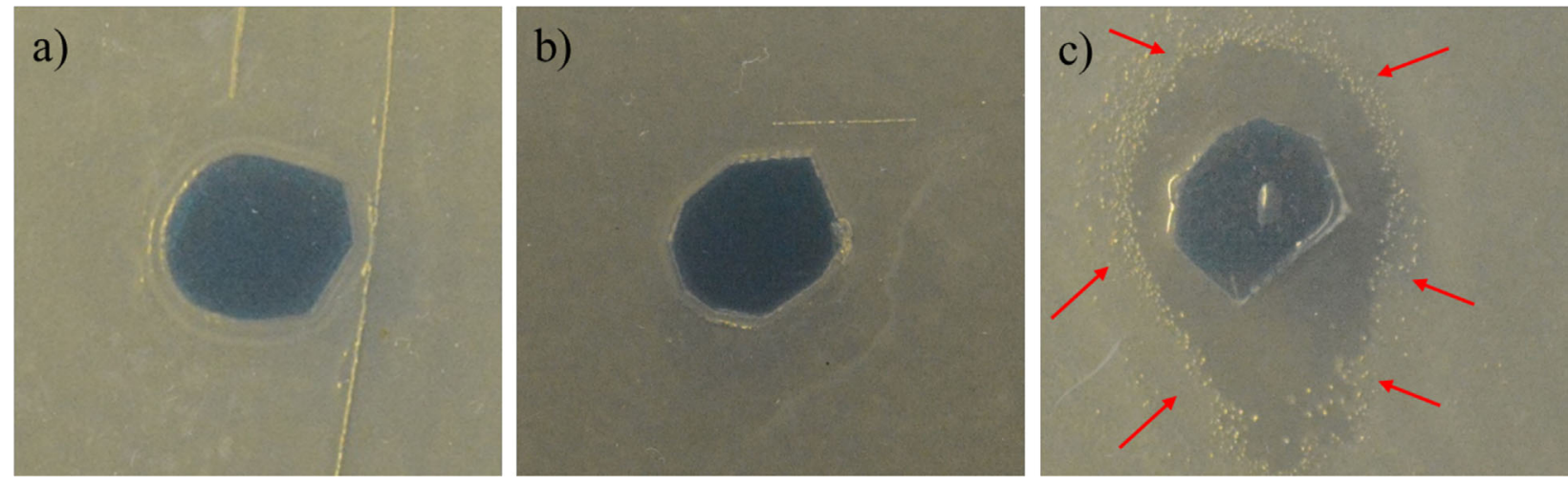

Figure 9. E. coli growth inhibition zones on TSA plates with uncoated PE (a), pPAA coated PE (b), and 5\% AgNPs/pPAA coated PE (c). 
Acknowledgements: This work was partially supported by the Italian Ministry of University and Research (MIUR), by the PORFESR grant (Regione Emilia Romagna), by the PLASMAT project (Alma Mater Studiorum-Università di Bologna, FARB grant) and by COST Action MP1101 Biomedical Applications of Atmospheric Pressure Plasma Technology and COST Action TD1208 Electrical Discharges with Liquids for Future Applications. The authors would like to acknowledge Daniela Barbieri for her contribution in antibacterial assay and Andrea Merlettini for his contribution in SEM/EDS analysis.

Supporting Information is available from the Wiley Online Library or from the author.

Received: July 29, 2015; Revised: September 23, 2015; Accepted: October 18, 2015;DOI: 10.1002/ppap.201500143

Keywords: Ag nanoparticles and pPAA co-deposition; antimicrobial properties; non-equilibrium atmospheric pressure plasma jet; SEM/EDS; XPS

[1] S. Kango, S. Kalia, A. Celli, J. Njuguna, Y. Habibi, R. Kumar, Prog. Polym. Sci. 2013, 38, 1232.

[2] S. Li, M. M. Lin, M. S. Toprak, D. K. Kim, M. Muhammed, Nano Rev. 2010, 1, 5214.

[3] I. Y. Jeon, J. B. Baek, Materials 2010, 3, 3654.

[4] G. Schmidt, M. M. Malwitz, Curr. Opin. Colloid Interface Sci. 2003, 8, 103.

[5] M. Groenewolt, M. Antonietti, Adv. Mater. 2005, 17, 14.

[6] A. A. Voevodin, J. S. Zabinski, Compos. Sci. Technol. 2005, 65, 741.

[7] Z. Guo, K. Lei, Y. Li, H. W. Ng, S. Prikhodko, H. T. Hahn, Compos. Sci. Technol. 2008, 68, 6.

[8] C. Aymonier, D. Bortzmeyer, R. Thomann, Rolf Mülhaupt, Chem. Mater. 2003, 15, 4874.

[9] Z. H. Mbhele, M. G. Salemane, C. G. C. E. van Sittert, J. M. Nedeljkovic, V. Djokovic, A. S. Luyt, Chem. Mater. 2003, 15, 5019.

[10] C. J. Weng, J. Y. Huang, K. Y. Huang, Y. S. Jhuo, M. H. Tsai, J. M. Yeh, Electrochim. Acta 2010, 55, 8430.

[11] X. Shi, T. A. Nguyen, Z. Suo, Y. Liu, R. Avci, Surf. Coat. Technol. 2009, 204, 237.

[12] Y. Li, L. Tang, J. Li, Electrochem. Commun. 2009, 11, 846.

[13] H. Biederman, Surf. Coat. Technol. 2011, 205, S10.

[14] H. Biederman, O. Kylian, M. Drabik, A. Choukourov, O. Polonskyi, P. Solar, Surf. Coat. Technol. 2012, 211, 127.

[15] H. Biederman, L. Holland, Nucl. Instr. Meth. 1983, 212, 497.

[16] U. Schurmann, H. Takele, V. Zaporojtchenko, F. Faupel, Thin Solid Films 2006, 515, 801.

[17] D. Ross, K. K. Gleason, Chem. Vap. Deposition 2006, 12, 225.

[18] X. Hou, K. L. Choy, Chem. Vap Deposition 2006, 12, 583.

[19] P. Marchand, I. A. Hassan, I. P. Parkin, C. J. Carmalt, Dalton Trans. 2013, 42, 9406.

[20] F. Fanelli, F. Fracassi, Plasma Chem. Plasma Process. 2014, 34 , 473.

[21] P. Heyse, R. Dams, S. Paulussen, K. Houthoofd, K. Janssen, P. A. Jacobs, B. F. Sels, Plasma Process Polym. 2007, 4, 145.

[22] G. Borcia, N. M. D. Brown, J. Phys. D: Appl. Phys. 2007, 4, 145.

[23] I. P. Vinigradov, A. Lunk, Surf. Coat. Technol. 2005, 200, 695.

[24] F. Palumbo, P. Favia, A. Rinaldi, M. Vulpio, R. d'Agostino, Plasmas Polym. 1999, 4, 133.
[25] A. J. Beck, R. D. Short, A. Matthews, Surf. Coat Technol. 2008, 203, 822.

[26] P. Cools, E. Sainz-Garcia, N. De Geyter, A. Nikiforov, M. Blajan, K. Shimizu, F. Alba-Elias, C. Leys, R. Morent, Plasma Process. Polym. 2015, doi: 10.1002/ppap.201500007.

[27] R. Morent, N. De Geyter, S. Van Vlierberghe, A. Beaurain, P. Dubruel, E. Payen, Prog. Org. Coat. 2011, 70, 336.

[28] M. Donegan, D. P. Dowling, Surf. Coat. Technol. 2013, 234, 53.

[29] O. Carton, D. B. Salem, S. Bhatt, J. Pulpytel, F. Arefi-Khonsari, Plasma Process. Polym. 2012, 9, 984.

[30] D. B. Salem, O. Carton, H. Fakhouri, J. Pulpytel, F. ArefiKhonsari, Plasma Process. Polym. 2014, 11, 269.

[31] A. Vogelsang, A. Ohl, R. Foest, K. Schroder, K. D. Weltmann, J. Phys. D: Appl. Phys. 2010, 43, 485201.

[32] G. Chen, M. Zhou, Z. Zhang, G. Lv, S. Massey, W. Smith, M. Tatoulian, Plasma Process. Polym. 2011, 8, 701.

[33] J. Bardon, J. Bour, D. Del Frari, C. Arnoult, D. Ruch, Plasma Process. Polym. 2009, 6, S655.

[34] A. Dembele, M. Rahman, I. Reid, B. Twomey, J. M. Don MacElroy, D. P. Dowling, J. Nanosci. Nanotechnol. 2011, 11, 1.

[35] A. Uygun, L. Oksuz, A. G. Yavuz, A. Gulec, S. Sen, Curr Appl Phys. 2011, 11, 250.

[36] M. Michel, J. Bour, J. Petersen, C. Arnoult, F. Ettingshausen, C. Roth, D. Ruch, Fuel Cells 2010, 10, 932.

[37] O. Feng, J. Wu, G. Chen, F. Cui, T. Kim, J. Kim, J. Biomed. Mater. Res. 2000, 52, 662.

[38] O. Beier, A. Pfuch, K. Horn, J. Weisser, M. Schnabelrauch, A. Schimanski, Plasma Process. Polym. 2013, 10, 77.

[39] X. Deng, C. Leys, D. Vujosevic, V. Vuksanovic, U. Cvelbar, N. De Geyter, R. Morent, A. Nikiforov, Plasma Process. Polym. 2014, 11, 921.

[40] H. R. Humud, A. K. Abass, Z. Wheeb, Asian J. Appl. Sci. Eng. 2014, 3, 25.

[41] A. Liguori, A. Pollicino, A. Stancampiano, F. Tarterini, M.L, Focarete, V. Colombo, M. Gherardi, Plasma Process. Polym. 2015, doi: 10.1002/ppap.201500080.

[42] M. Boselli, V. Colombo, E. Ghedini, M. Gherardi, R. Laurita, A Liguori, P. Sanibondi, A. Stancampiano, Plasma Chem. Plasma Process. 2014, 34, 4.

[43] M. Boselli, V. Colombo, E. Ghedini, M. Gherardi, R. Laurita, A. Liguori, P. Sanibondi, E. Simoncelli, A. Stancampiano, IEEE Trans. Plasma Sci. 2015, 43, 3.

[44] V. Colombo, D. Fabiani, M.L. Focarete, M. Gherardi, C. Gualandi, R. Laurita, M. Zaccaria, Plasma Process. Polym. 2014, 11, 247.

[45] V. Colombo, E. Ghedini, M. Gherardi, R. Laurita, P. Sanibondi, D. Fabiani, M. Zaccaria, M. L. Focarete, C. Gualandi, Proceedings of the 11th IEEE international conference on solid dielectrics - ICSD 11 2013, 358.

[46] D. Fabiani, M. Zaccaria, M. L. Focarete, C. Gualandi, V. Colombo, E. Ghedini, M. Gherardi, R. Laurita, P. Sanibondi, Proceedings of the 11th IEEE international conference on solid dielectrics - ICSD 11 2013, 718.

[47] X. Zhu, F. Arefi-Khonsari, C. Petit-Etienne, M. Tatoulian, Plasma Process. Polym. 2005, 2, 407.

[48] R. Morent, N. De Geyter, S. Van Vlierberghe, P. Dubruel, C. Leys, E. Schacht, Surf. Coat. Technol. 2009, 203, 1366.

[49] D. T. Clark, H. R. Thomas, J. Polym. Sci.: Polym. Chem. Ed. 1977, 15, 2843.

[50] M. Bashir, S. Bashir, Plasma Chem. Plasma Process. 2015, 35 , 739.

[51] P. Bosso, F. Fanelli, F. Fracassi, Plasma Process. Polym. 2015, doi: 10.1002/ppap.201500005. 
[52] P. Devaraj, P. Kumari, C. Aarti, A. Renganathan, J. Nanotechnol. 2013, 2013, 598328.

[53] T. Elavazhagan, K.D. Arunachalam, I. J. Nanomedicine 2011, 6, 1265.

[54] S. Bhatt, J. Pulpytel, S. Mori, M. Mirshahi, F. Arefi-Khonsari, Plasma Process. Polym. 2013, 11, 24.

[55] N. D. Boscher, F. Hilt, D. Duday, G. Frache, T. Fouquet, P. Choquet, Plasma Process. Polym. 2015, 12, 66.

[56] T. C. Kaspar, T. Droubay, S. A. Chambers, P. S. J. Bagus, J. Phys. Chem. C 2010, 114, 21562.

[57] A. V. Naumkin, A. Kraut-Vass, S. W. Gaarenstroom, C. J. Powell, NIST Standard Reference Database 2012, 20, Version 4.1 (http://srdata.nist.gov/xps/Default. aspx).

[58] C. N. Lok, C. M. Ho, R. Chen, O. Y. He, W. Y. Yu, H. Sun, P. H. Tam, J. F. Chiu, C. M. Che, J. Biol. Inorg. Chem. 2007, 12, 527.

[59] C. D. Wagner, Electron spectroscopy in: D. A. Shirley (Ed.), Proceedings of an International Conference, Asilomar, Pacific
Grove, California, USA, September 7-10, 1971, North-Holland, Amsterdam, 1972.

[60] C. D. Wagner, Anal. Chem. 1972, 44, 967.

[61] S. W. Gaarenstroom, N. Winograd, J. Chem. Phys. 1977, 67, 3500 .

[62] D. Briggs, M. P. Seah, Practical Surface Analysis, 2nd edition, John Wiley \& Sons, New York 1992.

[63] C. D. Wagner, W. M. Riggs, L. E. Davis, J. F. Moulder, G. E. Muilenberg, in Handbook of X-Ray Photoelectron Spectroscopy, Perkin-Elmer Corporation, Physical Electronics Division, Eden Prairie, MN 1979.

[64] N. K. Vu, A. Zille, F. R. Oliveira, N. Carneiro, A. P. Souto, Plasma Process. Polym. 2013, 10, 285.

[65] A. Sadeghnejad, A. Aroujalian, A. Raisi, S. Fazel, Surf. Coating Tech. 2014, 14, S0257.

[66] C. Damm, H. Münstedt, Appl. Phys. A 2008, 91, 479.

[67] X. Deng, A. Y. Nikiforov, T. Coenye, P. Cools, G. Aziz, R. Morent, N. De Geyter, C. Leys, Sci. Rep. 2015, 5, 10138. 